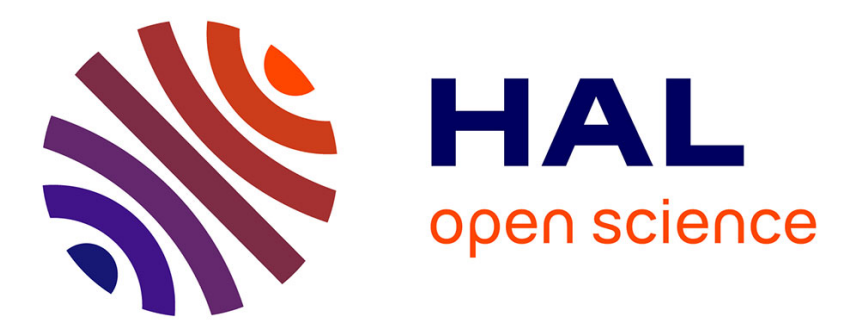

\title{
Les Nouveaux outils de l'aménagement forestier : l'exemple des systèmes d'information géographique.
}

Philippe Lejeune, Jacques Rondeux

\section{To cite this version:}

Philippe Lejeune, Jacques Rondeux. Les Nouveaux outils de l'aménagement forestier: l'exemple des systèmes d'information géographique.. Revue forestière française, 1999, 51 (sp), pp.169-184. 10.4267/2042/5494. hal-03443612

\section{HAL Id: hal-03443612 https://hal.science/hal-03443612}

Submitted on 23 Nov 2021

HAL is a multi-disciplinary open access archive for the deposit and dissemination of scientific research documents, whether they are published or not. The documents may come from teaching and research institutions in France or abroad, or from public or private research centers.
L'archive ouverte pluridisciplinaire HAL, est destinée au dépôt et à la diffusion de documents scientifiques de niveau recherche, publiés ou non, émanant des établissements d'enseignement et de recherche français ou étrangers, des laboratoires publics ou privés. 


\title{
LES NOUVEAUX OUTILS DE L'AMÉNAGEMENT FORESTIER : L'EXEMPLE DES SYSTĖMES D'INFORMATION GÉOGRAPHIQUE
}

\author{
P. LEJEUNE - J. RONDEUX
}

La gestion des milieux forestiers implique la maîtrise d'une quantité sans cesse croissante et diversifiée d'informations se caractérisant avant tout par leur composante spatiale. La cartographie joue donc depuis très longtemps un rôle important en foresterie. L'apparition, au début des années 1980, d'outils de gestion et d'analyse de données géoréférencées a généré de très nombreuses initiatives et applications dans le domaine de la cartographie forestière comme dans celui plus global de la gestion des territoires (Joliveau, 1996a). Ces réalisations ne répondent cependant pas toujours à des objectifs précis et reposent rarement sur une méthodologie clairement identifiée, ce qui peut se traduire dans certains cas par des échecs retentissants (Pornon, 1992) ou donner lieu à des applications peu convaincantes en termes de productivité et de rapport coût / bénéfice.

La formidable expansion que connaît actuellement, dans le domaine informatique, le créneau des applications géographiques, combinée à la diversité des objectifs et des moyens (humains et techniques) à la disposition des gestionnaires forestiers justifie de faire le point sur les possibilités d'utilisation de la cartographie numérique ou plus généralement de la géomatique dans le domaine forestier.

C'est dans ce contexte que s'inscrit le présent article dont la finalité est triple:

- présenter très brièvement les principales caractéristiques d'un système d'information géographique (SIG) et en décrire les composantes ;

- évoquer, au travers de quelques exemples concrets, les possibilités de valorisation de cet outil pour résoudre des problèmes d'aménagement, de gestion ou encore d'exploitation forestière ;

- présenter quelques orientations quant à la mise en place d'un SIG à vocation forestière, en insistant sur les questions les plus importantes à se poser et les éventuels pièges à éviter.

Nous terminerons cette présentation en évoquant les grandes tendances en matière d'évolutions technologiques dans le domaine des SIG et leurs retombées possibles pour les utilisateurs dans le domaine forestier. 


\section{P. LEJEUNE - J. RONDEUX}

\section{QU'EST-CE QU'UN SIG ?}

Un système d'information géographique est un système de gestion de base de données informatisé assurant la saisie, le stockage, l'extraction, l'interrogation, l'analyse et l'affichage de données localisées (Laurini et al., 1993). Si beaucoup d'utilisateurs de SIG assimilent ceux-ci uniquement à des logiciels et du matériel informatique, plusieurs auteurs s'accordent pour étendre la définition des SIG non seulement aux données qui sont manipulées (Didier et Bouveyron, 1993), mais également aux personnes qui les manipulent, voire aux principes et méthodes mis en œuvre dans ces systèmes (Therialut, 1995, cité par Joliveau, 1996a). Cette difficulté de définir précisément la notion de SIG explique sans doute pourquoi on associe le plus souvent ceux-ci à la seule composante "logiciel" dans laquelle apparaissent clairement les quatre grandes fonctionnalités de cet outil que sont :

- la saisie,

- la gestion, le stockage,

- la visualisation, l'édition,

- et, enfin, le traitement et l'exploitation d'une information spatialement référencée.

Avant de présenter ces fonctions, il est sans doute utile de décrire rapidement la manière avec laquelle les données sont représentées et structurées dans un SIG.

\section{Représentation des données dans un SIG}

La représentation des données au sein d'un SIG concerne à la fois les données spatiales relatives à la localisation et à la forme des objets dans l'espace et les données descriptives proprement dites, ou données thématiques, précisant les caractéristiques propres des mêmes objets : composition d'un peuplement, revêtement d'une route... II existe deux modes principaux de structuration de l'information dans un SIG : le mode vectoriel, aussi qualifié de mode objet, et le mode matriciel que l'on nomme également mode raster ou mode image (Collet, 1992). Le mode vectoriel repose sur une description numérique et géométrique explicite des objets distribués dans l'espace (Peuquet, 1990), ceux-ci relevant de trois types que sont les points, les lignes et les surfaces (figure 1a, ci-dessous). La représentation matricielle construit pour sa part une image du territoire

Figure 1

REPRÉSENTATION AU SEIN D'UN SIG DES DONNÉES SPATIALES

(a) EN MODE VECTORIEL ET (b) EN MODE RASTER :

exemple d'un objet surfacique (une parcelle) et d'un objet linéaire (un ruisseau)

(a)

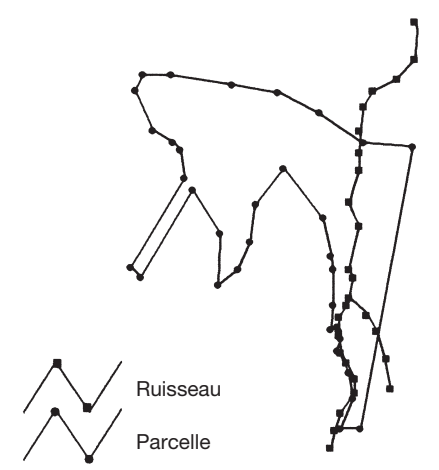

(b)

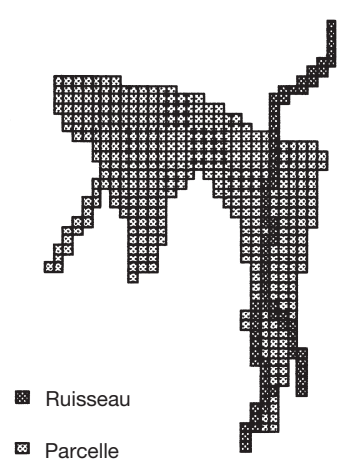


au travers d'une grille découpant l'espace en cellules (pixels) pouvant prendre chacune une valeur en fonction de la thématique considérée dans la carte (figure 1b, p. 170).

Chaque mode présente des avantages et des inconvénients (Burrough, 1986). Le mode vectoriel permet une exploitation directe des relations géométriques existant entre objets présents dans un même espace (proximité, contiguïté, voisinage). La maîtrise par le logiciel de ces relations, qualifiées de topologiques, est une condition nécessaire à la capacité que celui-ci aura d'analyser et d'exploiter de manière efficace les informations spatiales contenues dans la base de données. Cette maîtrise permet de différencier les logiciels de type "SIG" des logiciels "DAO" (dessin assisté par ordinateur) parfois utilisés pour des applications de cartographie numérique mais qui ne sont pas conçus pour des tâches d'analyse de données (Cowen, 1990). Une autre spécificité du mode vectoriel est la séparation physique qu'il opère entre informations spatiales et informations descriptives, celles-ci pouvant être gérées par un système de gestion de base de données classique (SGBD), indépendamment du logiciel SIG mis en œuvre.

La notion de topologie n'apparaît pas explicitement dans le mode matriciel. Celui-ci présente l'avantage d'une compatibilité avec les données issues de la télédétection satellitale qui, dans certaines applications forestières, est un support d'information de première importance. Ce mode matriciel est en outre doté d'une structure plus simple que celle du mode vectoriel, rendant les logiciels qui l'utilisent moins coûteux. Notons enfin qu'il est particulièrement bien adapté aux problèmes d'analyse impliquant des phénomènes à variation spatiale continue (Eastman, 1996) tels que l'altitude, la distance, la propagation d'un polluant ou d'un incendie de forêts par exemple.

\section{La saisie des données}

Bien que la saisie des données soit identifiée comme une fonctionnalité à part entière des SIG, elle n'est pas pour le forestier une fin en soi, mais bien le passage obligé permettant l'utilisation ultérieure du système. La phase d'acquisition constitue sans aucun doute l'étape la plus coûteuse dans la mise en œuvre d'un SIG, celle-ci pouvant représenter jusqu'à 80 \% du coût total du projet.

D'une manière générale, l'acquisition de l'information utilisée dans un SIG peut s'envisager selon trois voies (Brown, 1995) : digitalisation d'une carte, utilisation de documents photographiques et saisie d'informations spatiales in situ.

\section{- Digitalisation d'une carte "papier" préexistante}

II s'agit de la démarche mise en œuvre lorsque l'information existe sous la forme de carte "papier". On fait l'hypothèse que le géoréférencement des objets y est correct et que le contenu thématique correspond à ce qui est recherché. La digitalisation d'une carte des peuplements forestiers ou encore d'une carte pédologique, par exemple, peut être effectuée selon cette voie.

Cette digitalisation peut s'opérer manuellement à l'aide d'une table à digitaliser ou selon une procédure plus ou moins automatisée (Gerebtzoff, 1996), afin de réduire la durée des opérations qui varient fortement selon la complexité de la carte et, dans une moindre mesure, selon la dextérité de l'opérateur.

\section{- Utilisation de photographies aériennes ou d'orthophotoplans}

Lorsqu'on doit réaliser une cartographie de parcelles forestières ou de peuplements sans disposer de document préexistant, la photographie aérienne constitue sans doute la source d'information la plus adéquate pour atteindre cet objectif. II faut cependant être attentif à ce que celle-ci réponde à plusieurs critères : une échelle compatible avec le degré de détail recherché (1/10 000 à 1/25 000 


\section{P. LEJEUNE - J. RONDEUX}

selon le degré de morcellement de la forêt) ; une émulsion adaptée, idéalement l'infrarouge couleur ou la couleur vraie (Greer et al., 1990) et enfin une couverture qui soit la plus récente possible.

L'utilisation dans un SIG des informations spatiales saisies sur photographies aériennes nécessite l'application de corrections liées aux déformations géométriques induites par la topographie et les caméras utilisées. Ces corrections s'appuient sur la connaissance des caractéristiques de la caméra (focale) et du relief des zones photographiées (modèle numérique de terrain) ainsi que sur le calage des photographies dans le référentiel utilisé. Les photographies ainsi corrigées produisent des orthophotoplans ou orthophotographies (Manzer, 1995). Le développement de logiciels permettant la production de ces documents avec une précision et un coût acceptables constitue un progrès important pour la valorisation des photographies aériennes dans un SIG (Kersten et Chuat, 1997). Une approche plus simple, retenue par certains auteurs, consiste à digitaliser les limites de peuplements forestiers sur des photographies non redressées, et de n'appliquer les corrections géométriques qu'aux seuls points issus de cette digitalisation (Wolsack et Falcone, 1997).

Un autre problème doit être surmonté lors de l'utilisation de photographies aériennes : celui de l'interprétation des documents, permettant de caractériser les entités cartographiées. Cette démarche implique une bonne connaissance de terrain et conduit généralement l'opérateur à compléter cette phase de digitalisation sur photographie par une campagne de mesures-vérifications in situ.

\section{- Saisie des informations spatiales sur le terrain}

En l'absence de cartes de peuplements préexistantes ou de photographies aériennes adaptées, la saisie des données spatiales sur le terrain reste la seule solution envisageable. Celle-ci peut s'opérer par des moyens d'arpentage classique (clinomètre, boussole, topofil), moyennant le calage des objets levés par rapport au référentiel utilisé dans le SIG (utilisation de la carte topographique de référence). II s'agit d'une opération très exigeante en moyens humains. Elle peut dans certains cas mettre en œuvre des techniques plus modernes : système de positionnement universel (GPS), ordinateur de terrain... Bien que le milieu forestier présente certaines limites pour une mise en œuvre optimale de ces outils, liées essentiellement à l'interception par le couvert forestier d'une partie des signaux transmis par les satellites dans le cas du GPS (Lejeune et Hellemans, 2000) ou des conditions climatiques dans le cas de certains ordinateurs ou autres appareils de saisie électroniques, il est possible d'en tirer parti pour améliorer la productivité des opérations d'arpentage à des fins de cartographie forestière.

L'importance du coût d'acquisition des données pour la mise en place d'un SIG est renforcée par le fait que le système ne devient réellement opérationnel qu'à partir du moment où l'ensemble des données a été saisi. Cette contrainte, combinée au manque d'expérience en matière de digitalisation lors de l'installation du SIG, peut conduire l'entreprise à sous-traiter la saisie des fonds de plans initiaux, de manière à disposer rapidement d'une base de données opérationnelle.

\section{Gestion et stockage}

La gestion des données spatiales selon un des deux modèles présentés ci-avant (vectoriel et matriciel) se traduit par la création de couches d'informations, chaque couche se rapportant à un ensemble d'objets concernant un thème donné. La caractéristique première de la gestion des données dans un SIG est de rendre ces couches superposables dès lors qu'elles ont été définies dans le même référentiel géographique (figure 2, p. 173), celui-ci s'appuyant sur les notions de datum (modèle de représentation du globe terrestre) et de système de projection (ESRI, 1994). Cette nécessité de gérer les données spatiales dans un même référentiel géographique impose de s'assurer que toutes les cartes "papier" utilisées ont été produites avec le même système de projection ou de procéder, dans le cas contraire, aux corrections qui s'imposent (Lejeune, 1995). Ces corrections devront être appliquées notamment aux coordonnées produites par les GPS qui sont le 

DÉFINIES DANS UN MÊME RÉFÉRENTIEL GÉOGRAPHIQUE

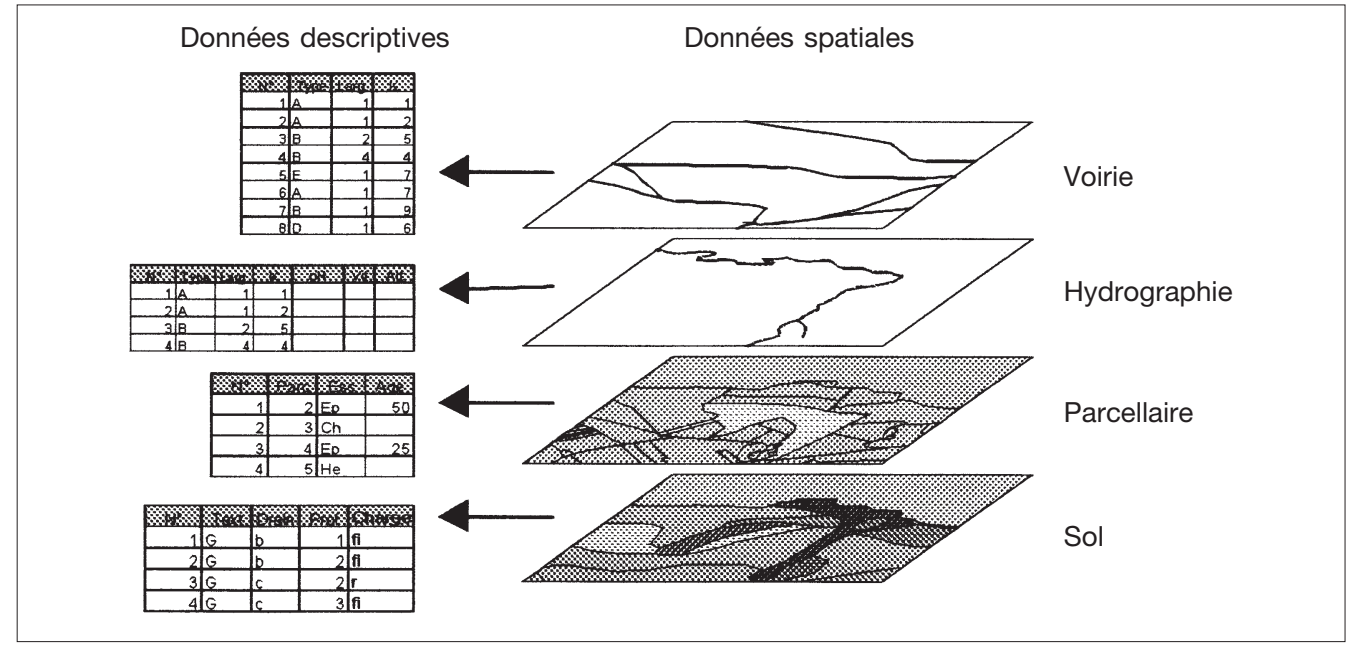

plus souvent exprimées dans le système de référence WGS84 (World Geodetic System), alors que la plupart des cartes utilisées en Belgique, par exemple, sont établies dans le système de coordonnées Lambert 72, propre à l'Institut géographique national de Belgique (IGN, 1989).

Les capacités de stockage et les périphériques de sauvegarde disponibles actuellement tendent à minimiser le problème du volume des données à stocker dans un SIG, qu'elles soient structurées en mode vectoriel ou en mode matriciel.

\section{Visualisation et édition}

Les fonctionnalités graphiques de visualisation et d'édition sont sans doute celles qui impressionnent le plus les utilisateurs de SIG débutants. Elles font du SIG un outil de communication particulièrement intéressant et ce d'autant plus que les documents cartographiques produits deviennent accessibles à d'autres utilisateurs (réseau Internet), peuvent être imprimés selon une infinité d'échelles (dans les limites de validité des données d'origine) sur des périphériques de bonne qualité ou encore récupérés dans une filière bureautique traditionnelle (illustration de rapports).

\section{Traitement et exploitation}

On peut envisager trois manières d'exploiter les données dans un SIG préalablement à leur édition (Pornon, 1992) : l'extraction, l'interrogation et l'analyse. L'extraction se fonde sur l'organisation en couches et sur leurs propriétés spatiales. Elle permet d'extraire de la base de données des groupes d'objets répondant à certains critères prédéterminés (par exemple extraire la carte des sols pour le "secteur nord" d'une propriété forestière). L'interrogation consiste à identifier dans la base de données un objet ou un groupe d'objets répondant à une requête (interrogation) utilisant des critères spatiaux ou thématiques (par exemple rechercher, dans un massif forestier, toutes les plantations d'épicéas de moins de 15 ans qui sont bordées par un cours d'eau).

Les fonctions d'analyse combinent des procédures d'extraction, de recherche, mais aussi de calcul. Celles-ci mettent en œuvre des opérateurs de type géométrique (inclusion, intersection, calcul de surface et de distance) exploitant le plus souvent les relations topologiques entre objets pour la création de zones tampons, l'intersection de couches ou encore la recherche du chemin optimal... 


\section{P. LEJEUNE - J. RONDEUX}

Ces fonctions d'analyse constituent les outils les plus intéressants sinon les plus puissants offerts par un SIG et, paradoxalement, elles sont aussi les moins utilisées.

\section{À QUOI PEUT SERVIR UN SIG ?}

Si l'on dépasse le plan théorique des fonctionnalités qui viennent d'être présentées et qu'on s'intéresse aux services que peut rendre un SIG au gestionnaire d'un territoire forestier, il est possible de mettre en évidence une certaine progressivité dans les missions qui lui sont assignées : collecte et inventaire de données, analyse et combinaison de ces données et, enfin, aide à la décision (Joliveau, 1996b). Dans les paragraphes qui suivent, nous présenterons un certain nombre d'exemples concrets d'utilisation d'un SIG dans le cadre de la gestion d'un massif boisé d'environ 400 ha. Ces exemples tentent de mettre en évidence le degré plus ou moins élaboré du traitement réalisé à l'aide du SIG.

\section{Liaison carte de gestion - parcellaire}

Le rôle de collecte et de compilation des données constitue le niveau élémentaire d'exploitation du SIG. II est particulièrement intéressant pour le forestier dans la mesure où plusieurs couches d'informations vont l'intéresser au premier plan (Jolly et Guyon, 1993) : assiette des parcelles, réseau de voirie, réseau hydrographique, sol, relief...

Cette compilation permet également la représentation cartographique d'une quantité importante d'informations descriptives des parcelles : âge et composition des peuplements, caractéristiques dendrométriques, programme des travaux... La liaison entre la couche contenant les limites des entités cartographiées et les tables de données descriptives peut s'opérer dans les deux directions : de la couche cartographique vers les tables descriptives (consultation de la fiche parcellaire d'une parcelle sélectionnée à l'écran) ou des tables vers la couche cartographique (production d'une carte thématique concernant, par exemple, l'âge des peuplements). L'édition de cartes thématiques "papier", à des échelles variables, portant sur tout le massif ou sur certaines parties de celuici et combinant une ou plusieurs couches d'informations, constitue un outil de travail très précieux pour le forestier et ce d'autant que les fonctions d'édition des objets dans les couches d'informations lui permettent de disposer de documents "papier" régulièrement et facilement mis à jour.

\section{Croisement de couches}

Au-delà du simple stade de la consultation des données, interviennent des fonctions de traitement pouvant porter sur les données spatiales, thématiques ou sur les deux types simultanément. Une autre distinction peut être faite selon que ces procédures mettent en œuvre une seule ou plusieurs couches d'informations.

Le croisement de couches est sans doute un des traitements les plus typiques et les plus démonstratifs dans un SIG. Celui-ci permet de définir la superposition géométrique des objets présents dans deux couches distinctes. Cette procédure est généralement opérée entre couches contenant des objets "surfaciques". Elle est particulièrement intéressante lorsque la couche contenant le parcellaire est croisée avec les cartes décrivant certains éléments du milieu, tels que le sol ou encore le relief. II en résulte une description de chaque parcelle par rapport à ces éléments ; description importante à considérer au moment de l'aménagement forestier par exemple. Cette description est non seulement de nature quantitative (quels types de sols sont présents dans la parcelle $x$ et en quelles proportions ?), mais également spatiale (comment se distribuent ces sols dans la parcelle ?). 
Figure $3 \quad$ CROISEMENT DE LA CARTE DES SOLS AVEC LE PARCELLAIRE FORESTIER

pour déterminer la proportion de sols à drainage déficient des unités de gestion (sous-parcelles) :

(a) carte des sols et (b) classement des sous-parcelles

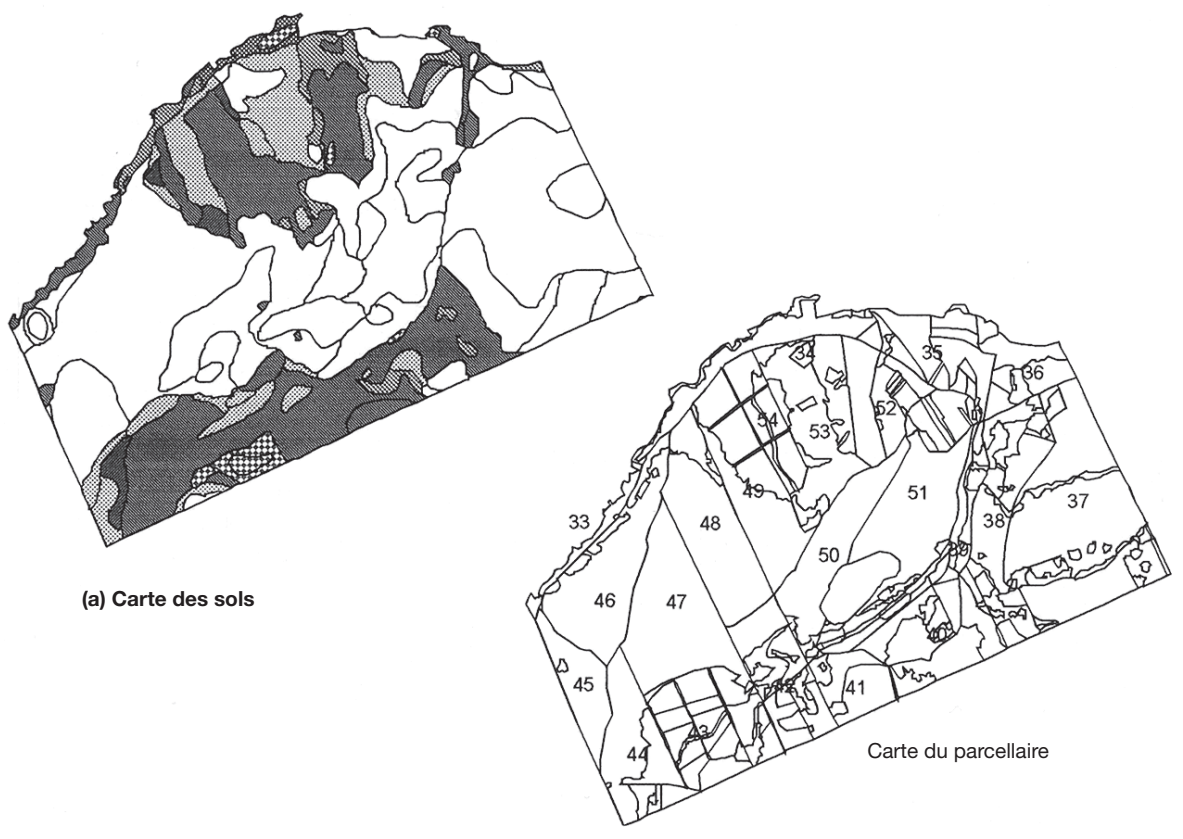

(b) Carte du drainage par sous-parcelles

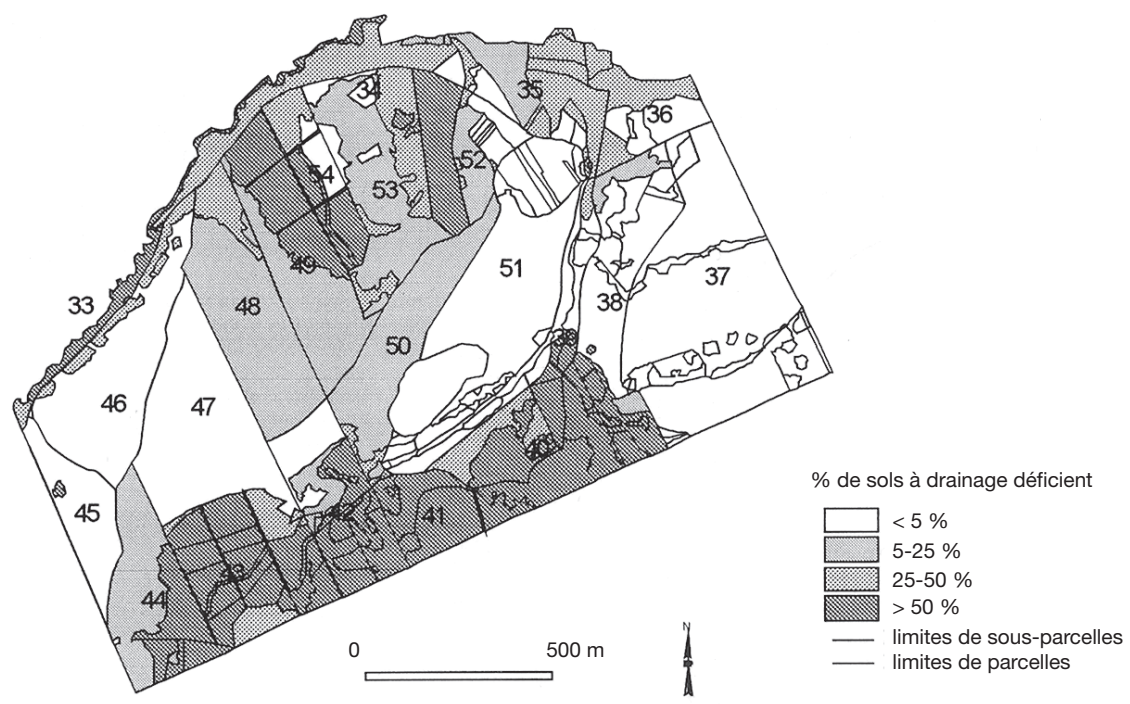




\section{P. LEJEUNE - J. RONDEUX}

La figure 3 (p. 175) montre un exemple de croisement de la couche "parcellaire" avec la carte des sols. II concerne la proportion de sols à drainage déficient présents dans chaque sous-parcelle.

Un autre croisement intéressant pour le forestier combine le parcellaire avec une couche constituée de points représentant des placettes d'inventaire par échantillonnage (de type aléatoire ou systématique). Un premier intérêt de cette application réside dans la conception du plan d'échantillonnage : le calcul du nombre d'unités d'échantillonnage en fonction du positionnement et de la dimension de la maille, la réalisation d'une stratification avec taux d'échantillonnage variable deviennent des opérations très simples dès lors que les grilles d'inventaire sont générées par le SIG et peuvent être croisées avec le parcellaire. La superposition de la grille qui est finalement retenue avec une carte de base (topographique) dans le SIG permet également de simplifier la préparation des opérations de cheminement (calcul des distances et azimuts des centres de placettes par rapport à des points remarquables). Finalement, lors du traitement et de l'exploitation des données, le SIG offre des possibilités de regroupement des unités d'échantillonnage selon d'autres critères que les limites de parcelles (possibilité de redécoupage des parcelles).

\section{Notion de distance}

Les croisements de couches qui viennent d'être présentés ne reposent que sur la simple notion de superposition géométrique d'objets. La prise en compte de la distance entre objets (d'une même couche ou de couches différentes) ouvre de nombreuses possibilités d'analyse intéressantes dans les problèmes d'environnement : impact des nuisances (Joerin, 1998), déplacement de la faune (localisation des zones de nourrissage et de remise), protection de zones sensibles (Bousson et al., 1998)... ou de valorisation des ressources notamment forestières : dimensionnement du réseau de voirie (Cheret et Gay, 1995), transport des matières premières vers les lieux de transformation (Emeyriat, 1997)... Cette distance peut être envisagée sous une forme simple ou de manière plus élaborée en intégrant un facteur de contrainte, la pente par exemple (Berry, 1993).

\section{Sectorisation du massif forestier}

L'étape qui suit l'exploitation et l'analyse des données dans un SIG est l'établissement de lignes directrices pouvant servir d'appui aux décisions que doit prendre le gestionnaire forestier. Ces orientations adoptent le plus souvent la forme de réponses à des questions du type "que va-t-il se passer si ... ? " ou " quels sont les endroits les plus aptes pour entreprendre telle mesure ? ». Ces applications mettent en œuvre des procédures d'analyse spatiale de même que des outils de modélisation s'imbriquant selon des schémas plus ou moins complexes.

Un exemple relevant de cette démarche concerne la sectorisation ou le zonage de l'espace forestier. Celle-ci a pour objectif de différencier dans le massif des vocations prioritaires relatives à la protection des zones sensibles, à la conservation des formations forestières de haute valeur biologique, sylvicole ou génétique et à la production ligneuse (Nivelle, 1990). Cette sectorisation repose à la fois sur une description des peuplements (structure, composition), du milieu (sol, hydrographie, relief, végétation) et sur la prise en compte des impératifs de gestion (homogénéité et dimension minimale des parcelles). L'analyse débouche sur une classification semi-automatique des parcelles, seuls les cas limites étant laissés à l'appréciation du gestionnaire (Bousson et al., 1998). L'intérêt de la méthode réside dans la possibilité de tester différentes valeurs-seuils pour la fixation des différents critères de sensibilité et de simuler l'impact de ces paramètres sur la configuration du parcellaire.

Les quelques exemples qui viennent d'être présentés montrent combien la mission d'un SIG peut varier selon qu'il est attaché à de simples applications de gestion ou à des démarches d'aide à la décision ou d'aménagement. Si l'expertise des personnes amenées à gérer le système doit être à la mesure de la complexité des tâches qui lui sont assignées, la fréquence de réalisation de ces 
opérations est par contre souvent inversement proportionnelle à cette même complexité : les cartes thématiques simples peuvent être produites annuellement (mise à jour du parcellaire) ou selon les besoins (suivi de travaux, mise en vente de coupes), alors que les cartes de synthèse et d'analyse ne sont élaborées que lors de la révision des aménagements (périodicité de 12 ans ou plus).

\section{COMMENT METTRE EN PLACE UN SIG À VOCATION FORESTIÈRE ?}

Si les aspects techniques jouent un rôle important dans la mise en place d'un SIG à vocation forestière, les aspects méthodologiques, économiques, humains et organisationnels ne doivent pas être occultés par les seules considérations de choix de "machines" et de logiciels.

\section{Fixer les objectifs}

Les premières questions à se poser ont trait aux objectifs poursuivis et concernent avant toute autre chose la pertinence d'implantation d'un tel système, cette question valant surtout pour les propriétés de petites dimensions $(<100 \mathrm{ha})$.

Si les objectifs du SIG se traduisent par les trois niveaux d'utilisation évoqués dans le paragraphe précédent (collecte, analyse et aide à la décision), il faut être conscient qu'une certaine "maturation" est nécessaire avant de pouvoir passer d'un niveau au niveau supérieur (Joliveau, 1996b). On peut même considérer que la plupart des applications restent cantonnées à des activités relevant du premier, voire du second niveau. Cependant, pour les projets qui ambitionnent d'atteindre le niveau d'aide à la décision, il est important d'évaluer les possibilités d'évolution du système tel qu'il est configuré initialement, tant au niveau du matériel que des logiciels.

\section{Identifier les données à récolter}

L'étape sans doute la plus importante dans la conception du projet est de fixer le niveau de détail retenu pour la cartographie de base du massif forestier. Ce choix a en effet un impact direct sur l'effort à consentir non seulement pour l'acquisition des données initiales, mais aussi pour leur mise à jour. Si ce niveau peut aller jusqu'à la délimitation des peuplements dans le cas de massifs de faible étendue ou de composition relativement homogène, il faut admettre que, pour des propriétés de plusieurs centaines d'hectares parfois assez morcelées, il devient illusoire de vouloir cartographier tous les peuplements.

L'étape suivante concerne le choix de la filière de digitalisation des contours des entités à cartographier. Celle-ci doit, selon les circonstances, tirer parti de toutes les sources d'informations disponibles : cartes ou orthophotoplans existants, réalisation de relevés de terrain. On observera que, si cette opération est confiée à un sous-traitant, il est important d'établir un cahier des charges soulignant le niveau de précision requis, et de procéder à une réception des données digitalisées, en s'assurant du respect de ces exigences.

Le choix d'une échelle de référence découle directement des considérations émises lors des deux étapes précédentes, de même que le choix du référentiel cartographique qui sera généralement celui des cartes topographiques disponibles pour la région considérée.

Parallèlement à la digitalisation du parcellaire, il convient de faire l'inventaire des couches nécessaires à l'accomplissement des différentes missions dévolues au SIG et la forme sous laquelle elles doivent être intégrées dans la base de données. Par exemple, dans le cas de la pédologie, celle-ci peut être mise en œuvre sous la forme d'une couche vectorielle nécessitant la digitalisation coûteuse de la carte des sols. Une solution plus simple consiste à scanner et géoréférencer ce document. Si cette deuxième solution limite les possibilités d'exploitation à une simple interpréta- 


\section{P. LEJEUNE - J. RONDEUX}

tion par superposition de cette image avec les autres couches, elle est cependant beaucoup moins onéreuse et peut néanmoins s'avérer suffisante dans certaines applications.

\section{Choix du matériel et des logiciels}

Le choix du matériel porte sur l'ordinateur et sur les périphériques qui lui sont associés. On peut considérer que les micro-ordinateurs disponibles actuellement sur le marché (Pentium ${ }^{\circledR}$ III) et généralement leurs prédécesseurs (processeur type 486) présentent des performances suffisantes pour assurer le fonctionnement de la plupart des logiciels de SIG et pour accueillir les bases de données de projets d'envergure normale, voire importante. Ce type de machine présente aussi l'avantage de supporter les logiciels courants de gestion (tableurs) et de bureautique (traitement de texte), facilitant de ce fait la liaison entre ces outils devenus incontournables pour le forestier (gestion courante, comptabilité, courrier, rapports...) et le SIG.

Les périphériques de saisie se divisent en deux catégories : les tables à digitaliser (saisie en mode vectoriel) et les scanners (saisie en mode image). Pour les premières, le prix est également lié à la taille de l'appareil et varie entre 500 euros (1) pour une tablette A4 à environ 6000 euros pour une table A0. En ce qui concerne les scanners, le prix varie non seulement en fonction de la dimension et de la résolution, mais également selon que le scanner est capable ou non de traiter des documents en couleur. Ici, également, des appareils de petite dimension (A4) développés en grande série pour les applications bureautiques sont accessibles à bas prix (moins de 250 euros), alors que les scanners de plus grand format (A0) nécessitent des investissements plus importants qu'ils fonctionnent en niveaux de gris (environ 6000 euros) ou en couleurs (plus de 15000 euros).

Les périphériques de sortie (imprimantes) se différencient essentiellement par la dimension des cartes qu'ils peuvent produire et la qualité d'impression de celles-ci. Les produits de bas de gamme correspondent aux imprimantes "couleur" à jet d'encre de format A4 utilisées par ailleurs pour les applications bureautiques et leur prix dépasse rarement 250 euros. Les machines de plus grande dimension (A0), dont le prix est beaucoup plus élevé (à partir de 7500 euros) ne se justifient que dans des projets plus importants et lorsque la taille des documents à produire augmente. Au-delà du prix d'achat de ces périphériques, il ne faut pas oublier leur coût de fonctionnement qui n'est pas négligeable (plusieurs euros voire dizaines d'euros par carte imprimée selon le format et la qualité d'impression).

Les logiciels de SIG se différencient selon leur degré de sophistication et la diversité des tâches qu'ils peuvent accomplir. La classification que nous proposons porte sur trois catégories décrites dans le tableau I (p. 179) : logiciels de visualisation (Viewers), SIG de bureau (Desktop Mapping Systems - DMS) et SIG professionnels (SIG pro). Étant donné leurs fonctionnalités limitées, la visualisation ne présente qu'un intérêt limité, à moins qu'elle ne soit utilisée, dans le contexte d'une architecture client-serveur d'un projet de grande envergure, sur des postes dédiés spécifiquement à la consultation.

Les DMS sont beaucoup plus complets. Outre les fonctions d'affichage et de présentation graphique intégrées dans une interface rendant leur utilisation très simple, ils contiennent des procédures de requêtes thématiques et spatiales plus ou moins développées. Leur module de mise en forme et d'impression de documents cartographiques leur confère une très grande attractivité. Enfin, ils disposent généralement d'un module de digitalisation et de mise à jour de couches cartographiques au départ d'une table à digitaliser ou de la souris (digitalisation à l'écran avec une image raster en arrière-plan). Leur capacité à importer et à lire des données de formats divers est un élément important à considérer lorsque l'on envisage de recourir à des bases de données cartographiques existantes.

(1) 1 euro $=6,55957 \mathrm{FF}$. 
Tableau I

Classification des logiciels de SIG en trois catégories: viewers, desktop mapping systems (DMS) et SIG professionnels

\begin{tabular}{|c|c|c|c|}
\hline \multirow[b]{2}{*}{ Fonctionnalités } & \multicolumn{3}{|c|}{ Gammes de logiciels } \\
\hline & Viewers & DMS & SIG pro \\
\hline Visualisation & + & ++ & ++ \\
\hline Liaison base de données $\alpha$-num. . . . & $+/-$ & + & ++ \\
\hline Digitalisation $\ldots .$. & - & $+/-$ & ++ \\
\hline Édition, production de documents & $+/-$ & + & ++ \\
\hline Requêtes spatiales simples $\ldots \ldots \ldots$ & - & + & ++ \\
\hline Requêtes spatiales complexes ...... & - & $+/-$ & ++ \\
\hline Fourchette de prix (euros) & $0-500$ & $500-2500$ & $2500-25000$ \\
\hline
\end{tabular}

Degré de développement des fonctions:-: nul, +/-: faible; +: élevé; ++:très élevé.

Les SIG professionnels sont en réalité de grosses "boîtes à outils" constituées de multiples modules nécessitant d'être manipulés par un expert à moins qu'ils ne constituent le support, via un langage de programmation spécifique, d'une application importante construite sur mesure. On retrouve, dans ces logiciels de haut de gamme, des fonctions d'édition, de manipulation et d'analyse de données qui fonctionnent généralement sur des données structurées en mode vectoriel ou matriciel. La gestion des données thématiques est prise en charge par des fonctions spécifiques au logiciel ou, le plus souvent, par un système de gestion de base de données classique.

Le développement de projets importants comportant de nombreux postes de travail dédiés à des tâches de nature différente (digitalisation, gestion, traitement, consultation...) implique de combiner plusieurs logiciels relevant des trois catégories. II est alors important de s'assurer d'une compatibilité totale entre ces différents produits.

Pour être complet dans la présentation des logiciels, il convient de signaler le rôle croissant que jouent les serveurs de données géoréférencées dans des applications importantes développées en réseau intranet ou internet (Turner, 1999). Cette évolution dans les stratégies de développement informatique tend à centraliser non seulement les bases de données mais également les ressources en termes de logiciels sur des machines "serveurs". L'utilisateur exploite alors ces ressources au départ d'un micro-ordinateur équipé simplement d'un logiciel de navigation (de type Netscape ${ }^{\circledR}$ ou Explorer®) et connecté à un réseau (intranet ou internet).

Comme on peut le constater, la diversité de solutions techniques pour la mise en place d'un SIG est très grande et il est hasardeux d'en sélectionner une en particulier, sans bien maîtriser les aspects méthodologiques, organisationnels et économiques évoqués précédemment. À titre indicatif, on peut cependant présenter deux configurations-types correspondant à des exigences fort différentes (tableau II, p. 180). La première présente l'équipement minimal permettant le fonctionnement d'un SIG de manière autonome, c'est-à-dire qui soit capable d'assurer la saisie des données, la réalisation d'un nombre limité de traitements et de requêtes, ainsi que la préparation et l'édition de cartes thématiques de bonne qualité. Le prix de cette installation est d'environ 4000 euros, le logiciel représentant à lui seul la moitié de l'investissement.

La deuxième configuration correspond à un projet plus important. Elle comporte deux postes de travail, l'un étant dédié aux tâches d'acquisition et de gestion des données, de même qu'aux analyses les plus élaborées (utilisation du SIG professionnel), l'autre servant essentiellement à l'ex- 


\section{P. LEJEUNE - J. RONDEUX}

ploitation courante des données et à la production de cartes finalisées. Les périphériques y sont plus élaborés. Tout comme dans la première configuration, la part des logiciels constitue environ la moitié de l'investissement, mais à un niveau sept fois plus élevé (29 000 euros).

Tableau II Évaluation du coût (en euros ${ }^{\star}$ ) de l'équipement et des logiciels pour l'installation d'un SIG: configuration minimale (1) et configuration pour une application plus importante (2)

\begin{tabular}{|c|c|}
\hline Configuration 1 & Configuration 2 \\
\hline $1 \mathrm{PC} \ldots \ldots \ldots \ldots \ldots \ldots \ldots \ldots \ldots$ & 2 PC en réseau $\ldots \ldots \ldots \ldots \ldots \ldots 3250$ \\
\hline 1 Imprimante A4 & Imprimante A4 \\
\hline 1 Scanner A4 & Imprimante $\mathrm{AO}$ \\
\hline 1 Unité de sauvegarde & 1 Scanner A4 \\
\hline 1 Desktop Mapping System . . . . . . . 2000 & 1 Table à digitaliser $(\mathrm{A} 0)$ \\
\hline & 1 Unité de sauvegarde $\ldots \ldots \ldots$ \\
\hline & 2 Desktop Mapping Systems \\
\hline & 1 SIG professionnel+SGBD $\ldots \ldots \ldots 7500$ \\
\hline Total . . . . . . . . . . . . . . 4000 & Total . . . . . . . . . . . . . . . . . . . . 29000 \\
\hline
\end{tabular}

* 1 euro $=6,55957 \mathrm{~F}$.

\section{Formation du personnel}

Même s'il se pose dans tous les cas, le problème de formation du personnel sera d'autant plus aigu que l'application est importante et que les acteurs sont multiples.

L'expérience montre qu'il est primordial que le personnel d'une organisation, impliqué dans la mise en place d'un SIG, soit convaincu de l'utilité de ce dernier. Cette motivation est d'autant plus déterminante que le projet ne devient réellement opérationnel, donc utile, qu'après une phase d'acquisition de données souvent longue et fastidieuse. Lorsque le personnel est affecté aux tâches "SIG" de manière partielle ou ponctuelle, il faut veiller à ce qu'il pratique et manipule le système avec une fréquence suffisante pour rester opérationnel et efficace dans sa maîtrise technique des différents outils (matériels et logiciels).

\section{Rapport coût / bénéfice}

II est difficile, voire impossible, d'évaluer a priori la rentabilité d'une application SIG (Pornon, 1992). Cela ne signifie pas qu'il faille négliger l'analyse économique d'un tel projet, celle-ci devant prendre la forme d'un bilan prévisionnel des coûts de fonctionnement. Ce bilan peut alors intervenir au même titre que les arguments techniques dans l'évaluation de l'utilité et de la faisabilité du projet.

\section{ÉVOLUTION ET PERSPECTIVES}

À l'aube du troisième millénaire, il est hasardeux de prédire l'évolution technique que connaîtra le secteur des SIG dans les prochaines années, tant celui-ci est en perpétuelle et rapide évolution. On peut cependant mettre en évidence trois tendances qui influenceront vraisemblablement le développement de projets à vocation forestière. 


\section{Architectures plus ouvertes et plus flexibles}

La première évolution concerne l'architecture des systèmes qui se veut de plus en plus ouverte, tant au niveau des formats de données, que de l'intégration avec les systèmes de gestion de bases de données (Golay, 1997), les systèmes de dessin assisté par ordinateur (Schutzberg, 1998), ou avec n'importe quelle application informatique fonctionnant dans l'entreprise. Cette tendance devrait améliorer sensiblement la rentabilité des projets de grande envergure présentant des ramifications souvent complexes avec d'autres applications.

\section{Saisie de données géoréférencées sur le terrain}

Le développement d'ordinateurs portables de terrain (Tristram, 1995) couplés à un GPS ou à un appareil de télémesures (Liu, 1995 ; Laser Technology, 1997) ou, plus simplement, équipé d'un logiciel SIG intégrant des fonctions COGO (coordination de la géométrie) devrait permettre de faciliter la saisie des données sur le terrain. Ces équipements représentent cependant des investissements considérables (plusieurs milliers d'euros) et ne se justifient que dans le cadre de projets couvrant des surfaces importantes (plusieurs dizaines de milliers d'hectares).

\section{Augmentation des performances des DMS}

Les logiciels de la catégorie Desktop Mapping System qui, à l'origine, étaient essentiellement conçus pour la production de cartes thématiques simples, s'enrichissent progressivement de fonctions d'analyses jusqu'alors présentes dans les seuls SIG professionnels. Ces fonctions sont le plus souvent rassemblées sous forme de modules spécialisés (données en réseau, données matricielles, analyses d'image, visualisation en 3D...), ce qui permet aux utilisateurs d'ajuster au mieux les équipements en fonction des problèmes rencontrés.

\section{CONCLUSIONS}

Dans le contexte de la gestion des ressources forestières, le SIG ne constitue pas la panacée, mais plutôt un outil qui, bien utilisé, peut rendre d'énormes services. II faut cependant garder à l'esprit qu'il constitue une technologie impliquant des investissements importants et dont il faut toujours évaluer l'opportunité de mise en œuvre.

Dans bien des cas en effet, la petite taille des propriétés forestières privées (quelques hectares) est tout à fait incompatible avec l'investissement qu'il faut consentir pour l'acquisition de l'équipement minimal. Une solution s'offre cependant à ces petits propriétaires : le recours aux services proposés par des entreprises spécialisées dans des tâches de cartographie et qui peuvent dès lors amortir les frais de fonctionnement sur des surfaces suffisantes. Cette formule de groupement existe déjà pour d'autres aspects de la gestion forestière : achats groupés, exploitation, centralisation de lots de bois...

Dès lors que la propriété atteint une taille suffisante (au-delà d'une centaine d'hectares), l'existence éventuelle d'un micro-ordinateur au sein de l'entreprise diminue de manière significative l'investissement à consentir pour acquérir un SIG. II n'en reste pas moins vrai que l'investissement en temps (apprentissage et saisie des données) reste important et dissuade le plus souvent le propriétaire ou le gestionnaire de s'engager dans une telle démarche. Dans ce contexte, la solution réside dans la mise en place d'un projet aux objectifs initiaux limités, basé sur un logiciel simple à utiliser, l'acquisition de fond de plan initial par sous-traitance pouvant alors s'avérer judicieuse.

C'est sans doute dans le contexte de la gestion des forêts publiques que l'intérêt d'implanter un projet SIG est le plus grand. Les superficies concernées dans ce cas sont généralement impor- 


\section{P. LEJEUNE - J. RONDEUX}

tantes. La gestion est en outre le plus souvent pratiquée de manière centralisée par le service forestier, chaque entité administrative pouvant comporter plusieurs milliers d'hectares de superficies boisées. Les missions à remplir par ces forêts publiques sont également les plus diversifiées, imposant la réalisation d'aménagements intégrant autant que possible les différentes fonctions de la forêt (production ligneuse, chasse, protection et conservation des milieux naturels, récréation). Le caractère centralisé de cette gestion des forêts publiques se traduira par la mise en place d'un projet de grande envergure, tant par les surfaces concernées, que par le nombre de personnes impliquées et la diversité des objectifs poursuivis. Le principal défi à relever dans un tel projet est de bien mesurer les moyens nécessaires au fonctionnement du SIG (acquisition et mise à jour des données !) et de s'assurer que ceux-ci sont compatibles avec la disponibilité en ressources humaines du service forestier, tant du point de vue quantitatif (personnel suffisant) que qualitatif (formation adaptée aux tâches demandées).

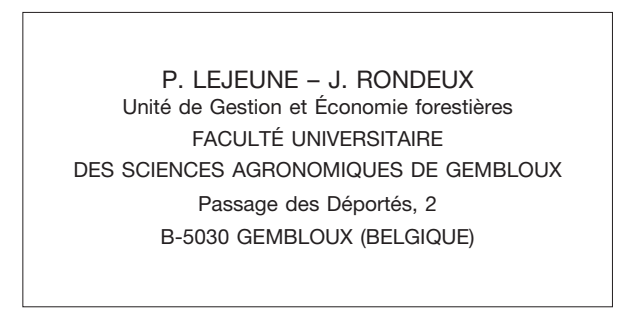

\section{BIBLIOGRAPHIE}

BERRY (J.K.). - Beyond mapping : concepts, algorithms and issue in GIS. - Fort Collins, Colorado : GIS World Books, 1993. - $246 \mathrm{p}$.

BOUSSON (E.), LEJEUNE (P.), RONDEUX (J.). - Application d'un SIG à la révision du parcellaire forestier dans le cadre d'aménagements forestiers intégrés. - Base, vol. 2, 1998, pp. 271-279.

BROWN (A.G.). - Discussion of three methods for creating GIS cadastral map layers. In : The GIS/LIS '95. Proceedings. Nashville, November 14-16, 1995. - Bethesda, Maryland : American Congress on Surveying and Mapping, 1995. - pp. 108-120.

BURROUGH (P.A.). - Principles of Geographical Information Systems for Land Resources Assessment. Oxford : Oxford University Press, 1986. - $193 \mathrm{p}$.

CHERET (V.), GAY (M.). - Utilisation d'un système d'information géographique pour l'élaboration d'un schéma de desserte ; exemple d'application sur le massif de Melles (Haute-Garonne). - Revue forestière française, vol. XLVII, n 6, 1995, pp. 647-660.

COLLET (C.). - Système d'information géographique en mode image. - Lausanne : Presses polytechniques et universitaires romandes, 1992. $-186 \mathrm{p}$. 


\section{L’aménagement forestier en pratique}

COWEN (D.J.). - GIS versus CAD versus DBMS : what are the differences ? In : Introductory readings in Geographic Information Systems / D.J. Peuquet and D.F. Marble Eds. - London : Taylor and Francis, 1990. - pp. 52-61.

DIDIER (M.), BOUVEYRON (C.). - Guide économique et méthodologique des SIG. - Paris : Hermès, 1993. $330 \mathrm{p}$.

EASTMAN (J.R.). - IDRISI for Windows. User's guide. - Worcester, Massachussets : Clark University, 1996.

EMEYRIAT (R.). - Intérêt des SIG pour améliorer la logistique de l'exploitation forestière. - Informations - Forêt, $\mathrm{n}^{\circ}$ 2-1997, fiche $\mathrm{n}^{\circ}$ 550, $6 \mathrm{p}$.

ESRI. - Map Projections. Georeferencing spatial data. - Redlands : Environnemental System Research Institute, 1994. - $236 \mathrm{p}$.

GEREBTZOFF (M.). - Problématique de la digitalisation de la carte des sols de Belgique : application à la Région wallonne. - Gembloux : Faculté universitaire des Sciences agronomiques, 1996. - 88 p. (Mémoire de fin d'études).

GOLAY (F.). - Architecture des systèmes d'information géographique. In : Séminaire "Orthophotos et réalité virtuelle en mensuration officielle", EPFL, 24-28 février 1997. - Lausanne : École polytechnique fédérale, 1997.

GREER (J.D.), HOPPUS (M.L.), LACHOWSKI (H.M.). - Color infrared photography for resource management. Journal of Forestry, 88, 1990, pp. 12-17.

IGN. - Systèmes de référence et formules de transformation en usage en Belgique. - Bruxelles : Institut géographique national, 1989. - $48 \mathrm{p}$.

JOERIN (F.). - Décider sur le territoire. Proposition d'une approche par utilisation de SIG et de méthodes d'analyse multicritère. - Lausanne : École polytechnique fédérale - Département de Génie rural, 1998. 220 p. + annexes (Thèse présentée pour l'obtention du grade de Docteur ès Sciences techniques).

JOLIVEAU (T.). - Gérer l'environnement avec des SIG. Mais qu'est-ce qu'un SIG ? - Revue géographique de Lyon, 71, 1996a, pp. 101-110.

JOLIVEAU (T.). - Éditorial : systèmes d'information géographique et gestion environnementale, de nouveaux outils pour des pratiques nouvelles ? - Revue géographique de Lyon, 71, 1996b, pp. 99-100.

JOLLY (A.), GUYON (D.). - Possibilités de suivi par un système d'information géographique de la gestion des forêts traitées en futaie régulière. - Revue forestière française, vol. XLV, numéro spécial "Informatique et Foresterie", 1993, pp. 108-121.

KERSTEN (T.), CHUAT (N.). - Le Projet swissphoto recouvre la Suisse en orthophotos. In : Séminaire "Orthophotos et réalité virtuelle en mensuration officielle”, EPFL, 24-28 février 1997. - Lausanne : École polytechnique fédérale, 1997.

LAURINI (R), MILLERET-RAFFORD (F.). - Les Bases de données en géomatique. - Paris : Hermès, 1993. $340 \mathrm{p}$.

LASER TECHNOLOGY INC. - 1997. - http://www.lasertech.com/laserproducts/geolaser.html

LEJEUNE (P.). - Carte des sols de Belgique et SIG : un traitement préalable visant à la concordance géométrique. - Bulletin des Recherches agronomiques de Gembloux, vol. 30, 1995, pp. 339-351.

LEJEUNE (P.), HELLEMANS (P.). - Effet du couvert forestier sur la précision d'un système de positionnement global différentiel (dGPS). - Biotechnology, Agronomy, Society and Environment, 4, 2000, sous presse.

LIU (C.J.). - Using portable laser EDM for forest traverse surveys. - Canadian Journal of Forest Research, vol. 25, 1995, pp. 753-766.

MANZER (G.). - Maximizing digital orthophoto use, a technical overview. - GIS World, vol. 8, $\mathrm{n}^{\circ} 12$, 1995, pp. 50-55.

NIVELLE (J.-L.). - De la gestion de la nature en milieu rural. In : Gérer la nature. Actes du colloque, Anseremme, 17-20 octobre 1989. - Namur, Belgique : Région Wallonne, 1990. - pp. 247-263.

PEUQUET (D.J.). - A conceptual framework and comparison of spatial data models. In : Introductory readings in Geographic Information Systems / D.J. Peuquet and D.F. Marble Eds. - London : Taylor and Francis, 1990. - pp. 250-285.

PORNON (H.). - Les SIG, mise en œuvre et applications. - Paris : Hermès, 1992. - 158 p.

SCHUTZBERG (A.). - CAD/GIS Integration : modern technology merges the best of both worlds. - GIS World, vol. 11, n 6, 1998, tiré de www.geoplace.com/print/gw/1998/0698/698mrtch.htm.

TURNER (A.K.). - Get your maps on the internet. - Geo World, vol. 12, 1999, pp. 32-33.

WOLSACK (J.), FALCONE (P.). - Une méthode cartographique d'inventaire forestier. - Nogent-sur-Vernisson : Inventaire forestier national, 1997. - 8 p. (Note technique). 


\section{LES NOUVEAUX OUTILS DE L'AMÉNAGEMENT FORESTIER : L'EXEMPLE DES SYSTÈMES D'INFORMA- TION GÉOGRAPHIQUE (Résumé)}

Que ce soit dans ses démarches d'analyse, de planification globale ou encore de gestion courante, le forestier est amené à collecter, traiter et utiliser une quantité importante de données présentant un caractère géoréférencé.

Avec l'avènement de l'informatique sont apparus des outils spécifiques de traitement de telles informations, communément appelés Systèmes d'Information Géographique (SIG). Le formidable développement de ces équipements, d'une haute technicité, n'a malheureusement pas toujours fait l'objet d'accompagnements méthodologiques appropriés permettant au forestier de terrain de tirer au mieux parti de ces outils.

Le présent article tente de faire le point sur les possibilités d'utilisation des SIG en gestion forestière. II met l'accent sur trois axes de réflexions : qu'est-ce qu'un SIG ; à quoi sert-il et comment le mettre en place?

\section{NEW TOOLS FOR FOREST PLANNING : THE EXAMPLE OF GEOGRAPHIC INFORMATION SYSTEMS (Abstract)}

Forest managers have to collect, process and use huge amounts of data for analysis, planning or management purposes. Most of these data have a spatial component which make them useful for decision-making.

The development of information technology has given rise to specific tools for the processing of spatial data : Geographic Information Systems (GIS). The methodological improvements required to take full advantage of these tools in practical applications have not always followed the development of this technology.

The main goal of this paper is to take stock of the potential for using GIS in forest management. The emphasis is on three questions : (i) what is a GIS, (ii) what is it useful for and (iii) how is it implemented? 